plus serrées qu'avant ; c'est là le résultat le plus sensible obtenu... peu de choses en somme.

A côté du service d'inspection gouvernemental, de nombreuses communes ont institué un service de contrôle municipal ; certains de ces services attachent la plus grande importance, je suis tenté d'écrire "trop d'importance " à l'essai de filtration et continuent à colporter, à afficher et à mettre en vedette dans des expositions le test de filtration "Avant l'inspection et après l'inspection ". Nous avons depuis quelques mois une " commission du lait $n$ comprenant toutes les compétences belges en matière de production laitière. S'occupera-t-elle de reviser les dispositions réglementaires que j'ai étudiées plus haut?

Comme je l'ai dit, la filtration doit être rendue obligatoire. C'est une question primordiale, mais il serait nécessaire d'avertir les consommateurs, aussi bien que les producteurs, que cette mesure $n^{\prime}$ a pas pour effet de transformer un lait infecté en un produit parfait.

Il est toujours dangereux d'entrer dans trop de détails, de vouloir trop préciser en matière de réglementation; le texte légal belge de 1894 n'aurait pas dû être modifié ; si on tenait absolument à le changer, on aurait pu adopter la rédaction française de 1924 qui spécifie, sans plus, que le lait sera recueilli proprement et qu'il ne sera ni coloré, ni malpropre, ni malodorant.

C'est clair et net, et les tribunaux ont ainsi une base solide pour la répression, l'exemple des jugements prononcés en France nous en donne la preuve.

\title{
UNE MÉTHODE RAPIDE POUR L'ESTIMATION DES PROPRIÉTÉS ÉLASTIQUES ET PLASTIQUES de la caséine a la présure
}

\author{
par \\ G. GENIN \\ Ingénieur Chimiste E. P. C.
}

On sait l'importance que présente la caséine en tant que matière première pour la fabrication de certaines matières plastiques, constituées essentiellement de caséine durcie à la formaldéhyde et qui sont connues en particulier sous le nom de galalith.

Il est certain que l'utilisation de ta caséine pour la fabrication de ces produits et l'emploi de ces derniers se sont trouvés limités du fait de la variabilité de la matière première utilisée. Autrement dit, lorsque dans une usine, on utilise des lots successifs de caséine à la présure pour fabriquer de la galalith ou toute substance analogue, on est parfois dans l'obligation d'apporter certaines modifications 
aux procédés de fabrication standards pour tenir compte des caractéristiques diverses des différents lots de caséine utilisés.

On voit done tout l'intérêt que pouvait présenter la mise au point d'une méthode d'essai permettant d'évaluer les propriétés plastiques et élastiques d'une caséine à la présure déterminée et de prévoir à l'avance dans quelles conditions il faudra traiter ce produit lors de sa transformation en matière plastique. Nous devons une étude de ce genre à C. A. Cooper (Brit. Plasties, mars 1939, p. 572) qui a décrit une méthode qui permet de comparer les propriétés élastiques et plastiques de caséine à la présure, méthode qui repose sur la compression, entre des plateaux chauffés, de pièces moulées de dimensions standards. Cet auteur a examiné de cette façon plus de 60 échantillons provenant de divers pays, et il a constaté qu'il n'existe pas, semble-t-il, de relation définie entre la déformation de ces caséines sous l'action de la chaleur et de la pression et les autres propriétés du produit telles que la teneur en graisse, en cendres, le $p \mathrm{H}$, etc. Il a pu également étudier par cette méthode l'influence d'un certain nombre de facteurs comme par exemple la température, la pression et la teneur en humidité de la caséine, sur sa déformation. De tous les essais ainsi effectués, il résulte que cette méthode d'essai présente un grand intérêt, car elle doit permettre au fabricant de caséine de parvenir à fournir un produit de qualité standard correspondant exactement aux exigences des fabricants de matières plastiques à base de caséine.

Tous eeux qui ont eu l'occasion de traiter la caséine à la présure sont parfaitement renseignés sur le fait que ce produit peut varier au point de vue plasticité et moulabilité. Par moulabilité, on peut définir la capacité d'un produit à conserver la forme qu'il a reçue sous l'action de la pression, lorsqu'on cesse d'exercer cet effort de pression. Or, à ce point de vue, les caséines d'une même origine sont parfois excessivement variables et il importe de prévoir à l'avance si un lot de caséine peut être considéré comme satisfaisant pour un usage industriel.

Au cours de son étude, Cooper s'est tout d'abord efforcé de définir une caséine standard, autant que cette définition soit possible avec un produit aussi variable que la caséine qui, comme toutes les protéines, diffère d'un échantillon au suivant. Pour préparer cet échantillon standard, l'auteur s'est réservé un certain nombre de sacs d'environ 50 kilogrammes chacun provenant d'un lot qui avait donné de bons résultats industriels. Ces sacs ont été parfaitement mélangés et mis de côté pour tous les essais, on ne broyait qu'un seul sac à là fois, le reste de la caséine étant conservé à l'état non broyé.

Pour procéder aux mesures de déformation, l'auteur a utilisé 
le plastomètre Scott, appareil de construction américaine et qui est bien connu de tous les fabricants de caoutchouc, car il est utilisé pour l'étude de la plasticité des mélanges de caoutchouc. L'appareil se compose essentiellement d'un plateau inférieur fixe et d'un plateau supérieur qui peut être élevé ou abaissé, le déplacement des deux plateaux l'un par rapport à l'autre pouvant être mesuré à 1 millième de pouce près. Ces plateaux sont creux, de façon qu'on puisse les chauffer par un courant d'eau chaude ou de vapeur et l'effort de pression s'exerce simplement en plaçant des poids sur une plate-forme fixée au plateau supérieur.

Dans les expériences de CoOPER, les plateaux étaient chauffés en faisant passer dans les canaux de circulation de l'eau chaude provenant d'un autoclave à chauffage électrique et dont la tempéraure était contrôlée au moyen d'un thermostat et maintenue rigoureusement égale à $120^{\circ}$. La circulation de l'eau était assurée par une pompe centrifuge et la température à l'intérieur des plateaux, mesurée par des thermomètres, était pratiquement identique à celle de l'eau à l'intérieur de l'autoclave, par suite de la circulation extrêmement rapide du fluide chauffant.

L'essai de déformation consistait essentiellement à comprimer une éprouvette cylindrique de dimensions standards entre les deux plateaux, la température et la pression étant rigoureusement déterminées. Les cylindres servant aux mesures se préparaient en mélangeant 100 grammes de caséine d'une teneur en humidité constante et égale à $12,7 \%$, avec $2 \mathrm{gr}$. 2 de plastifiant et $19 \mathrm{~cm}^{3}$ d'eau distillée. Lorsque l'humidité de la caséine utilisée s'écartait de la proportion de $12,7 \%$, on modifiait la quantité d'eau distillée ajoutée. La poudre humectée était comprimée dans un moule de construction spéciale, permettant d'obtenir simultanément 6 cylindres identiques et de démouler ces cylindres sans les déformer. Après moulage sous une presse hydraulique, le moule était ouvert, de façon à obtenir des eylindres ayant environ 9 millimètres de diamètre et 6 millimètres de hauteur. Les conditions de moulage étaient les suivantes : 30 minutes à $105^{\circ} \mathrm{C}$, , et 10 minutes à froid, la pression étant d'environ 1 tonne par pouce carré.

Un autre problème qui se posait était de conserver ces cylindres dans des conditions telles qu'aueune perte d'humidité ne se produise avant l'essai. A cet effet, les cylindres étaient placés dans une atmosphère dont la teneur en humidité était de $92,6 \%$ et maintenus à la température ordinaire.

Les essais ont été effectués dans l'appareil Scott, soit sur des cylindres tels qu'ils avaient été préparés par simple compression ou sur des cylindres durcis par le formol. Dans ce dernier cas, ces cylindres étaient découpés dans des baguettes durcies de 6 milli- 
mètres de diamètre, en petites longueurs de 6 millimètres dont les sections étaient soigneusement dressées. Les petits cylindres; durcis avant d'être essayés, étaient conditionnés pendant quatre jours dans une atmosphère maintenue à $25^{\circ}$ et à une humidité relative de $75 \%$.

Le tableau ci-dessous donne les conditions dans lesquelles était effectué l'essai de compression :

\begin{tabular}{|c|c|c|}
\hline & Eehantillon non formolé & Echantillon formolé \\
\hline Température des plateaux ..... & $81^{\circ} \mathrm{C}$. & $115^{\circ} \mathrm{C}$ \\
\hline Charge totale .............. & $4,42 \mathrm{~kg}$. & $-16 \mathrm{~kg}$ \\
\hline Diamètre du cylindre d'essai ... & $9 \mathrm{~mm}$ & $6 \mathrm{~mm}$. \\
\hline Pression sur le cylindre........ & $6,12 \mathrm{~kg} \cdot / \mathrm{cm}^{2}$ & $54,4 \mathrm{~kg} . / \mathrm{cm}^{2}$ \\
\hline $\begin{array}{c}\text { Durée de préchauffage sur les } \\
\text { plateaux } \ldots \ldots \ldots \ldots \ldots \ldots \ldots\end{array}$ & 1 min. & $1 \min$. \\
\hline
\end{tabular}

Pour effectuer l'essai, on commençait par mesurer très exactement au moyen d'un micromètre la hauteur des cylindres. On séparait alors les plateaux préalablement chauffés d'une distance égale à cettehauteur et on plaçait le cylindre entre les deux plateaux, de façon qu'il soit parfaitement à leur contact, mais sans être comprimé. On chauffait alors dans ces conditions le cylindre pendant exactement une minute et au bout de ce temps, on chargeait le plateau supérieur du poids voulu. Ce cylindre se déformait et on déterminait la diminution de hauteur en fonetion du temps, diminution qui était enregistrée avec soin. Au bout de 3 minutes, on relevait le plateau supérieur, on retirait le cylindre et après refroidissement, on mesurait à nouveau sa hauteur. D'après les mesures de la hauteur initiale, de la hauteur finale et de la hauteur à la fin de la période de 3 minutes de compression, on déterminait les caractéristiques suivantes:

Déformation temporaire. C'est la diminution de hauteur observée au bout de 3 minutes de compression et exprimée en pourcentage de la hauteur initiale, lorsque l'échantillon est soumis à une température et à une pression déterminées.

Déformation permanente. C'est la diminution de hauteur de l'échantillon, exprimée en pourcentage de la hauteur initiale, lorsque l'échantillon, après avoir été retiré de la presse et refroidi, est mesuré à nouveau.

Récupération. C'est la différence entre la déformation temporaire et la déformation permanente, différence que l'on peut considérer comme une mesure de l'élasticité de l'échantillon ou de sa résistance à la déformation.

Examinons maintenant les résultats qui ont été obtenus par 
CoOper, en faisant varier les conditions de l'essai, et l'intérét qu'il faut attacher aux résultats numériques obtenus.

\section{Variation de la déformation avec la teneur en humidité}

Les résultats obtenus montrent que dans le cas de la easéine non durcie, il existe une augmentation régulière de la déformation temporaire lorsque la teneur en humidité augmente. Dans le cas du produit formolé, il n'y a aucune relation de ce genre, ce qui montre combien est faible la similitude des propriétés entre la caséine non formolée et le produit qui résulte de l'action chimique de la formaldéhyde sur la molécule de caséine.

\section{Variation de la déformation avec la température}

Dans le cas de la caséine non formolée, la déformation s'élève presque linéairement en fonction de la température.

\section{Variation de la déformation avec la pression}

Dans le cas de la caséine non formolée, la déformation temporaire, comme également la déformation permanente, commencent par s'élever graduellement lorsque la compression augmente pour tendre vers une valeur limite de déformation, lorsque la pression continue à augmenter. La récupération, par contre, est presque indépendante de l'effort de compression exercé, et reste à peu près constante ou ne varie que très peu, autour d'une valeur moyenne.

\section{Variation des propriétés des caséines avec la contrée d'origine}

Pour étudier cette variation, Cooper a déterminé la déformation temporaire et la récupération de 60 échantillons provenant de sept pays différents. On a pu ainsi démontrer l'existence dans certains pays de caséines très peu plastiques, c'est-à-dire de produits qui présentent une faible déformation temporaire et une récupération élevée. Ces caséines sont très difficiles à boudiner et on voit donc que l'essai à l'appareil Scott permet de mettre rapidement en évidence un défaut de ce genre.

Les valeurs moyennes déterminées sur les caséines provenant de différents pays montrent que la plasticité de la caséine dépend jusqu'à un certain point de son origine. Il semble par exemple que l'Argentine, l'Australie et la Nouvelle-Zélande produisent des caséines dures et peu plastiques, alors que la Hollande, la Norvège et la France donnent des caséines qui appartiennent à la classe des produits plus mous. 


\section{Relation entre la déformation et les autres propriétés de la caséine}

Il n'a pas été possible d'établir des relations très nettes entre les déformations établies avec l'appareil Scott et certaines autres propriétés de la caséine, comme son $p \mathbf{H}$, sa teneur en graisse, en cendres, etc. Toutefois, il semblerait que les caséines dures sont celles qui, généralement, ont un $p \mathrm{H}$ et une teneur en cendres élevés, tandis que les caséines molles ont un $p \mathrm{H}$ et une teneur en cendres bas. Par exemple, un échantillon de caséine dure d'Argentine avait un $p \mathrm{H}$ de 7,85 et une teneur en cendres de $8,8 \%$, tandis qu'à l'autre extrémité une caséine très molle de Hollande avait un $p \mathrm{H}$ de $7,0 \%$ et une teneur en cendres de $7,4 \%$.

On voit done que les mesures effectuées au plastomètre constituent un moyen très utile d'appréciation de la caséine et complètent heureusement les indications données par l'examen physique et chimique. Il semble qu'une étude approfondie de la compressibilité des caséines à l'appareil Scott devrait permettre aux différents producteurs de caséine du monde de parvenir à assurer une plus grande uniformité des propriétés physiques du produit. Il est possible en effet qu'on parvienne à déterminer quels sont les facteurs qui interviennent sur les propriétés élastiques et plastiques de la caséine, par exemple : fraîcheur du lait écrémé, température de l'action de la présure, constitution du lait, soin apporté au lavage de la caillebotte, nature de l'eau de lavage, température et durée du séchage, etc. Quand on aura mis en évidence l'influence de ces différents facteurs, on peut espérer qu'on parviendra à établir les meilleures conditions pour la production d'une caséine se rapprochant $d u$ produit idéal pour la préparation des matières plastiques.

\section{REVUE}

\section{L'INDUSTRIE LAITIERE A L'ÉTRANGER}

par G. GÉNIN

Ingénieur Chimiste E. P. C.

ANGLETERRE

\section{Un nouveau véhicule réfrigéré pour la livraison de la crème glacée}

Une firme anglaise vient de concevoir un nouveau type de véhicule pour la livraison de la crème glacée. Ce véhicule est chargé 